\title{
Investigation of the Influence of Controller Types on Room Thermal Behaviour - A Simulation Study
}

\author{
Kristin Majetta ${ }^{1}$, Christoph Clau $\beta^{1}$, Christoph Nytsch-Geusen ${ }^{2}$ \\ ${ }^{1}$ Fraunhofer IIS EAS, Zeunerstraße 38, D-01069 Dresden, GERMANY \\ kristin.majetta@eas.iis.fraunhofer.de \\ christoph@clauss-it.com \\ ${ }^{2}$ Fachgebiet für Versorgungsplanung und Versorgungstechnik, Berlin University of the Arts, Hardenbergstr. 33, \\ D-10623 Berlin, GERMANY \\ nytsch@udk-berlin.de
}

\begin{abstract}
To control the indoor temperature of rooms two kinds of approaches are common. The first one is to use standard PI-controllers with a set of default parameters, which often leads to insufficient performance, waste of energy and unacceptable comfort violations [Rahmati, 2003]. The other approach is to use specifically developed and adapted controllers [Seidel et al., 2015], which have the drawback in a time-consuming and expensive development. Therefore, this paper investigates on finding rules and guidelines to find a suitable controller for a given room without the need of expensive controller adaption via simulation. To provide those rules a simulation study will be performed. This paper presents the first preparatory steps of this investigation, which includes the choice and development of four different room models equipped with different heating systems, which are an electrical radiator, a floor heating system, and a water supplied radiator. The authors present five types of controller models of different controller types to control the operative temperature of a room. Simulations of well-defined scenarios analyze the eligibility of the controller models regarding net energy consumption and comfort for the considered room models. First optimization results to improve the quality of the controllers are shown and further steps are explained.
\end{abstract}

Keywords: Building Simulation, Room Controller, Room Thermal Behavior, Optimization

\section{Introduction}

The German government plans the reduction of the primary energy consumption by $20 \%$ by the year 2020 compared to 2008 and, even more ambitious, by $50 \%$ by 2050. Many actions are taken to achieve this goal. One is the foundation and support of research projects to develop energy saving technologies. In the buildings sector one of these technologies are advanced control strategies to regulate e.g. indoor climate, energy consumption or the choice of an energy source out of different energy supplies. Normally, those controller strategies are developed and adapted to a specific room for which they work efficiently. Often those controllers can only be sufficiently adapted to other buildings under application of relatively large simulation effort which includes the model development and parameterization of the considered room, the comparison of the room behavior with measurements and the parameterization as well as an optimization of the controller model. Therefore, the adaption to other rooms or buildings is expensive and often the energy reduction is in no relation to the effort of adjusting the controller. This is why nowadays often basic controllers with default parameters are used which is not sufficient to achieve the control goals regarding room temperature and net energy consumption. During the last year's research in the field of indoor room temperature controllers, several example control algorithms have been developed. In [Lauenburg, 2014] for example, the control of a radiator heating system is optimized. A similar approach is presented in [Carlon, 2014] where the energetic performance of a low-energy house in analyzed and two possible control strategies of the biomass boiler heating systems are investigated. Very high research effort during the last years was done in the field of model predictive control methods where the future behavior of the room is predicted by simulating and optimizing a room model to calculate the in the future needed set points to ensure comfort and energy optimality. Deputizing for the abundance of research activities and literature in this field the following references are named: [Afram, 2014; Parisio, 2013; Oldewurtel, 2010; Ma, 2009]. A drawback of this method is that the needed prediction model must be developed which is normally done from measurements, and that this model needs to be updated after each optimization run. Rules would be helpful that support the choice of indoor room temperature controllers including a suitable set of parameters that fit best for the considered room. Therefore, a methodology is needed which provides those rules and guidelines for typical use cases of temperature controllers with regard to given rooms and their installed HVAC technology. This paper presents first steps of this investigation, which is the development of four different, representative room models as well as five controller models of important controller types. By means of simulations of defined scenarios suitable for each type of room (e.g. office room, class room), the eligibility of the controller 
models is investigated regarding the net energy consumption and the adherence by temperature comfort boundaries. First optimization results to improve the quality of the controllers are shown and further necessary steps are explained.

\section{Modeling}

\subsection{Building models}

To investigate the influence of different controller models on room behavior, four room models were built, that differ in size, building materials, heating system and usage. The chosen rooms are an office room, a class room a meeting room and a summerhouse (also called room since it consists of a single thermal zone). All models are equipped with different heating systems (electrical radiator, radiator flown through by water, floor heating) so that the typically range of room heating supply techniques is covered. That way it is assured that the methodology, which will be developed for choosing a suitable controller, is universally applicable and can easily be transferred to other problems.

The models of the four example rooms are built from model components of the Modelica BuildingSystems library, which is developed by the University of Arts (UdK), Berlin [Nytsch-Geusen, 2013]. This library can describe the behavior of complex building systems which consist of thermal and hygrothermal models of single buildings or districts in combination with the corresponding energetic supply techniques. The technical building services can contain thermal, hydraulic or electrical models for solar heat, photovoltaic, and HVAC systems. The room models developed for the purpose of this investigation are based on the Building1Zone1DBox-template that describes a single thermal zone with six opaque boundaries that can contain windows. The template is equipped with connectors compatible to the ambient model of the BuildingSystems library, with thermal ports to supply the building zone with heat and with a connector for the air change rate. The air temperature TAir of the zone is supplied by the model via an output connector. Figure 1 shows the graphical representation of the Building1Zone1DBox template connected to the ambient model.

heat port for convective heat heat port for longe-wave radiation

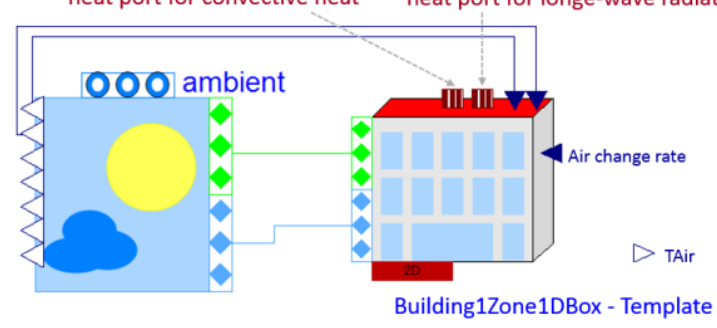

Figure 1: Template Building1Zone1D connected to ambient model
The ambient model provides the outside air temperature, the relative humidity of the ambient air, the wind speed and the solar radiation. The influence of the solar radiation on the operative room temperature depends mainly on the orientation of the windows, which are modelled within the Building1Zone1DBox template.

To assure the comparability of results, all room models receive the same ambient conditions from the TMY (typical meteorological year) [Deutscher Wetterdienst, 2014] of Chemnitz, a city in the east of Germany with approximately 250.000 inhabitants. For the sake of simplicity internal loads are not part of this study.

\section{Summerhouse}

The model of the summerhouse has a floor space of 30 $\mathrm{m}^{2}$, a height of $3.5 \mathrm{~m}$ and, deviating from Figure 2, it is modeled with six boundaries, which represent four walls, the ceiling and the floor.

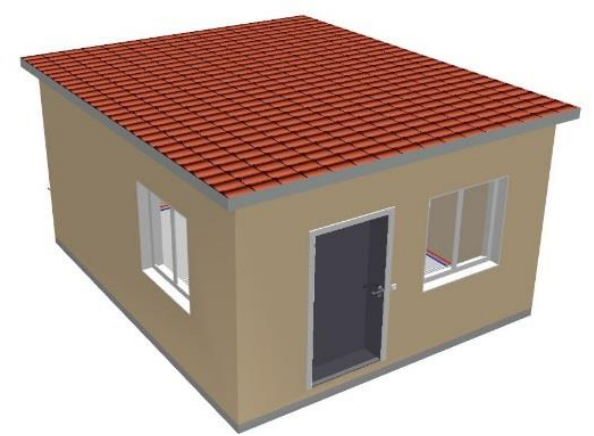

Figure 2: 3D representation of the summerhouse model

The summerhouse is modeled as a free-standing room, which means that the adjacent conditions of the boundaries (except for the floor) of the thermal zone are the ambient conditions. The adjacent boundaries are modeled as heavy construction from plastering, Styrofoam and bricks from concrete. The summerhouse model is equipped with a model of a $2 \mathrm{~kW}$ electrical heating system. The actuating mechanism of the heating system is discrete which means it can either be switched on or off completely. Therefore, the control signal provided by the heating controller must also be discrete.

\section{Single office room}

The model of the office room is suitable for one person. It has a floor space of $15 \mathrm{~m}^{2}$ and a height of $2.7 \mathrm{~m}$. It is enclosed by six boundaries of which the west oriented boundary adjacent conditions are the ambient conditions. The other boundaries border on neighbour rooms and have constant adjacent temperatures of 20 ${ }^{\circ} \mathrm{C}$. The west oriented boundary is modeled as heavy construction. The other materials are wood for the ceiling two boundaries (walls) that border on neighbour rooms and concrete for the wall that separated the office room from the floor. Figure 3 shows a picture of the office room that was used as a basis to develop the room model. 


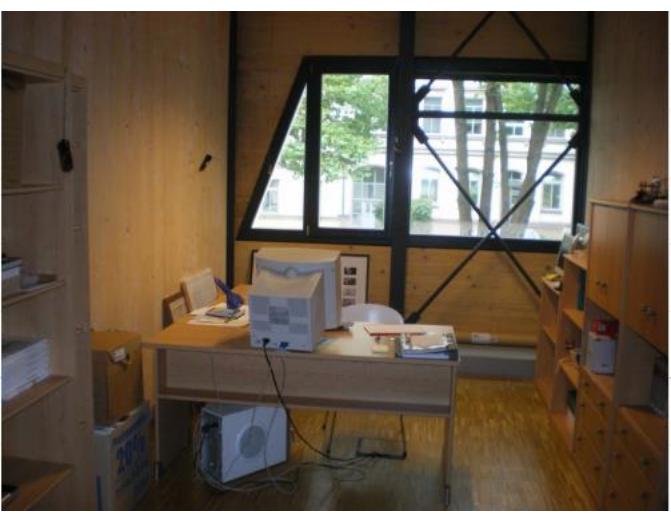

Figure 3. Picture of the office room on which the model is based

The office room model is equipped with a floor heating system that works with a supply temperature of $35^{\circ} \mathrm{C}$. The model of the heating system calculates a heating power $Q$ using (1). In (1) $T_{F}$ is the temperature of the floor surface, $T_{R}$ is the room temperature and $A$ is the floor area (Recknagel, 2012). $T_{F}$ is calculated from the supply temperature which is given to the heating system as input signal.

$$
Q=8.92 *\left(T_{F}-T_{R}\right)^{1.1} * A_{F}
$$

The heating power $Q$ is given to the room as input signal. The heating model has an input connector for its control signal. The required control signal must be Boolean. In case of control signal = true the heating system provides a heating signal of a certain amount of heat to the room model. If control signal $=$ false the amount of heat provided by the heating system is zero.

\section{Meeting room}

The model of the meeting room is based on a small conference room at Fraunhofer IIS/EAS. It is designed for meetings and workshops for about 20 people (Figure 4).

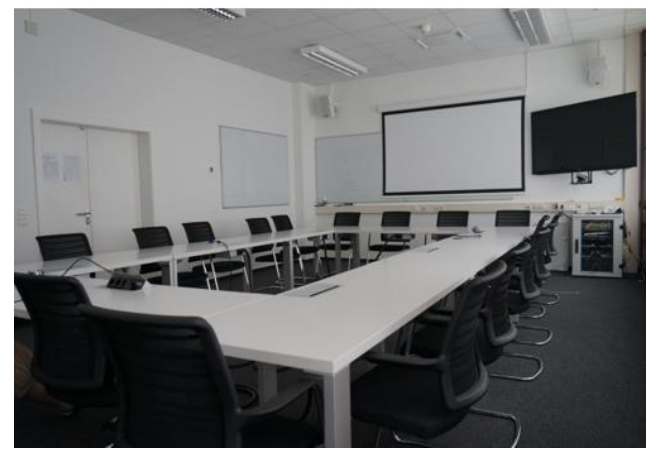

Figure 4. Picture of the meeting room on which the model is based

The room model has a floor space of $52 \mathrm{~m}^{2}$ and a height of $3.3 \mathrm{~m}$. it has one outer wall, the west oriented wall, at which ambient conditions are applied. The boundary temperature of the opposite wall and the ceiling is constant $18^{\circ} \mathrm{C}$, and for the other walls, it is 20 ${ }^{\circ} \mathrm{C}$. The four walls are modeled as heavy construction from clinker bricks and plastering. The ceiling and the floor are modeled from lightweight concrete. Also an interior ceiling is included which is made of papiermâché. The room model is equipped with a water heating systems (Figure 5) that gives radiation and convective heat via a radiator to the room model. The model of the water heating system, which is taken from the BuildingSystems library, is modelled as fluidic system. It contains a pump, pipes, a valve to regulate the volume flow, a radiator, an expansion vessel as well as a boiler. To regulate the volume flow of the water running through the radiator model, the valve model is controlled by its actuator position according to the valve characteristic, which specifies the volume flow rate depending on the valve position. Accordingly, the control signal calculated by a controller has to take values between 0 and 1 .

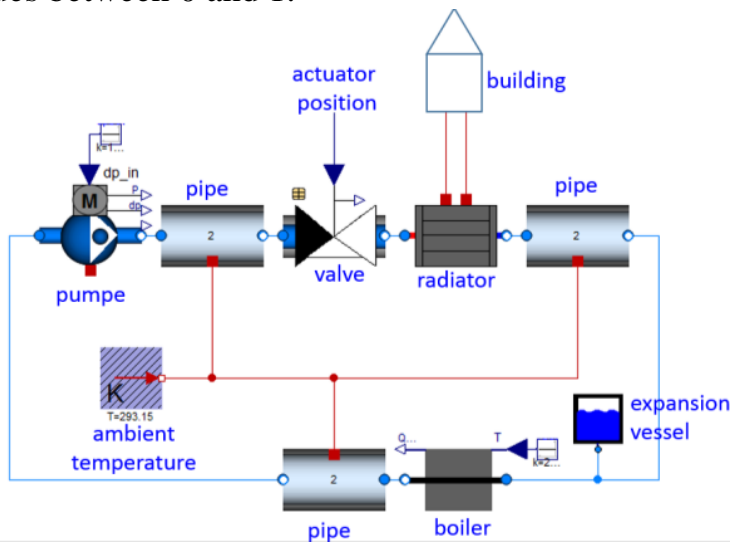

Figure 5. Water heating system

\section{Classroom}

The classroom model is based on data of the plus-energy primary school in Hohen Neuendorf near Berlin which was built as part of the research program Energieoptimiertes Bauen (EnOB) founded by the German Federal Ministry of Economic Affairs and Energy [Sick, 2015]. Figure 6 shows a picture of one classroom of the school, that was the basis for the classroom model.

The model has a floor space of $94 \mathrm{~m}^{2}$ and a height of $4 \mathrm{~m}$. It contains one outer wall that's boundary conditions are the ambient conditions.

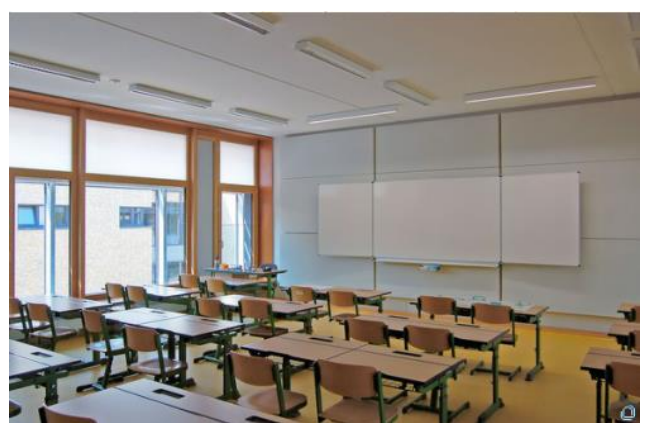

Figure 6. Picture of the classroom on which the model is based 
It is modeled as lightweight construction from wood, mineral wool and plasterboard. The inner walls are also lightweight and modeled from the materials wood and mineral wool. The floor consists of concrete, cement, bitumen, ethafoam and linoleum. The ceiling is modeled from concrete, cement and linoleum. The boundary temperatures of the inner walls are constant at $17^{\circ} \mathrm{C}$. The water based heating system is the same as described for the meeting room model.

\subsection{Controller models}

On the way of developing the methodology for choosing a suitable controller for a given room, the conformability of different controller models to the four introduced room models is investigated. Therefore, five common control strategies were chosen and modeled in Modelica. Partly they were developed in research projects at Fraunhofer IIS/EAS, partly they are taken from the Modelica Standard Library. The choice of the controller models can easily be extended. For example, the application of a model predictive controller is planned.

\section{Two-Point Controller}

The model of the two-point controller compares the actual room temperature with a required set point temperature. It provides a heating signal of 1 if the room temperature is below the set point temperature, which means the heating system should be turned on. The controller provides a heating signal of 0 if the room temperature is above the set point temperature. A hysteresis parameter prevents the controller from switching on and off permanently. This parameter influences the span between the given temperature set point and the actual temperature for turning the heating system on or off. Therefore, the height of the hysteresis influences the user comfort and the effective heating energy.

\section{Forward-looking Switching (FS)}

The aim of this controller is to determine the right moment to turn the heating system of a room model on and off to reach a desired target temperature at a specific point in time [Majetta, 2015]. Under the assumption of a given ambient temperature $T_{A}(t)$, a given supply temperature of a water heated heating system, respectively heating power in case of an electrical heating system $T_{S}(t)$, given temperatures of the adjacent rooms $T_{N}(t)$ and a given start value of the room temperature $T_{R}(t=0)$, the idea of this controller is to approximate the room temperature $T_{R}(t)$ by the response of a first order system $f(t)$ to a step change as first approximation. This is easily possible because the heating and cooling characteristics of single rooms are known neglecting disturbances and providing it with constant power. Figure 7 shows exemplarily the heating of the office room model under constant ambient and neighbour room temperatures.

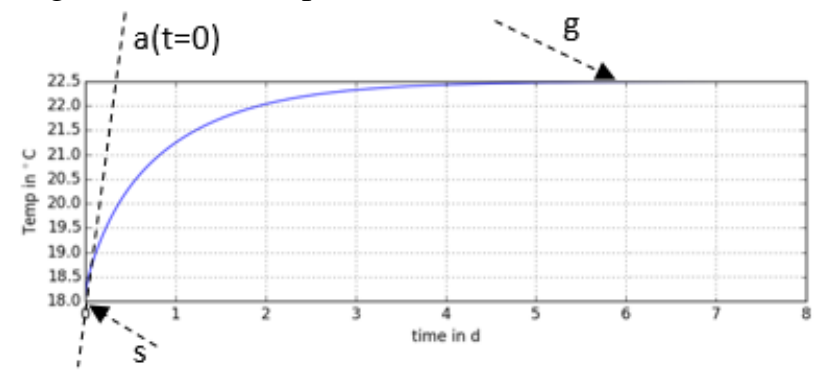

Figure 7. Heating up process of office room model

$f(t)$ is characterized by its steady-state value $g$, its start value $s$ and its slope of the temperature change $a(t=$ 0 ) at time $t=0$ and can be described by (2).

$$
f(t)=g-(g-s) e^{\frac{-a}{g-s} t}
$$

The variables $g$ and $a$ depend on $T_{S}(t), T_{R}(t=0)$, $T_{A}(t)$ and $T_{N}(t)$. Internal loads are not considered. For simplicity reasons the temperatures of the neighbour rooms are chosen to be identical. The variables $g$ and $a$ are identified using results from particular simulations with defined constant values of $T_{S}(t), T_{R}(t=0), T_{A}(t)$, and $T_{N}(t)$. In the following, the identification process of $g$ is shown exemplarily. To identify the dependency of the steady-state value $g$ from $T_{A}(t), T_{N}(t)$ and $T_{S}(t)$, the linear approach

$$
g=T_{A} \cdot x_{1}+T_{N} \cdot x_{2}+T_{S} \cdot x_{3}
$$

was chosen. To identify the unknowns $\left(x_{1}, x_{2}, x_{3}\right)$ in (3), $n$ simulations under defined conditions were undertaken and the linear system

$$
A x=b
$$

with

$$
A=\left[\begin{array}{ccc}
T_{A, 1} & T_{N, 1} & T_{S, 1} \\
\vdots & \vdots & \vdots \\
T_{A, n} & T_{N, n} & T_{S, n}
\end{array}\right] \text { and } b=\left[\begin{array}{c}
g_{1} \\
\vdots \\
g_{n}
\end{array}\right]
$$

is solved applying the least squares method [Isermann, 1991]. To do so, a solution $x^{*}$ shall be calculated that minimizes the quadratic error $\mathrm{err}=\left|A x^{*}-b\right|^{2} \cdot x^{*}$ is calculated by solving the normal equation $A^{T} A x^{*}=$ $A^{T} b$. Using (2) for each value of $T_{A}, T_{N}$, and $T_{S}, g$ can be calculated. A similar approach is applied for identifying the parameter $a$. The parameter $s$ does not have to be identified since it is the start value of the room temperature, which simply can be taken from the simulation. Knowing $g, s$ and $a(t=0)$, (2) is parameterized during the whole simulation of the room model and calculates the points in time for turning on or off the heating system online by transforming (1) to

$$
t_{o n, o f f}(t)=-\frac{g-s}{a} \ln \left(-\frac{w-g}{g-s}\right)
$$


where $w$ is the desired target temperature in the room. Since the heating and cooling process of the room model cannot be described exactly by an exponential function, two parameters were introduced to the controller model to optimize the point in time for turning the heating system on and off.

Combination of Two-point controller and Forwardlooking Switching - Combi-Controller

Both of the introduced controllers have properties that might be considered as drawbacks. The two-point controller responds to a change of the set point not before the change happened, i.e. in case of a desired temperature that is warmer than the actual set point, the heating system starts to warm up the room at that moment the desired room temperature should be already reached. That means that it probably, especially in the case of a slow working heating system will be too cold in the room for some time. Once the desired target temperature is reached, the two-point controller works well within the tolerance given by the hysteresis.

The forward-looking switching has the ability to turn the heating system on and off at the right moment in order to achieve a desired target temperature in the future. However, once it is turned on, no mechanism is available to prevent the room from overheating. In the upper part of Figure 8 the room temperature is shown that results from controlling the room temperature with the two-point controller (red continuous line marked with dots) and the forward-looking switching controller (blue dashed line). The desired target temperature is pictured as the green continuous line. The temperature related heating signal is pictured in the lower part of Figure 8.
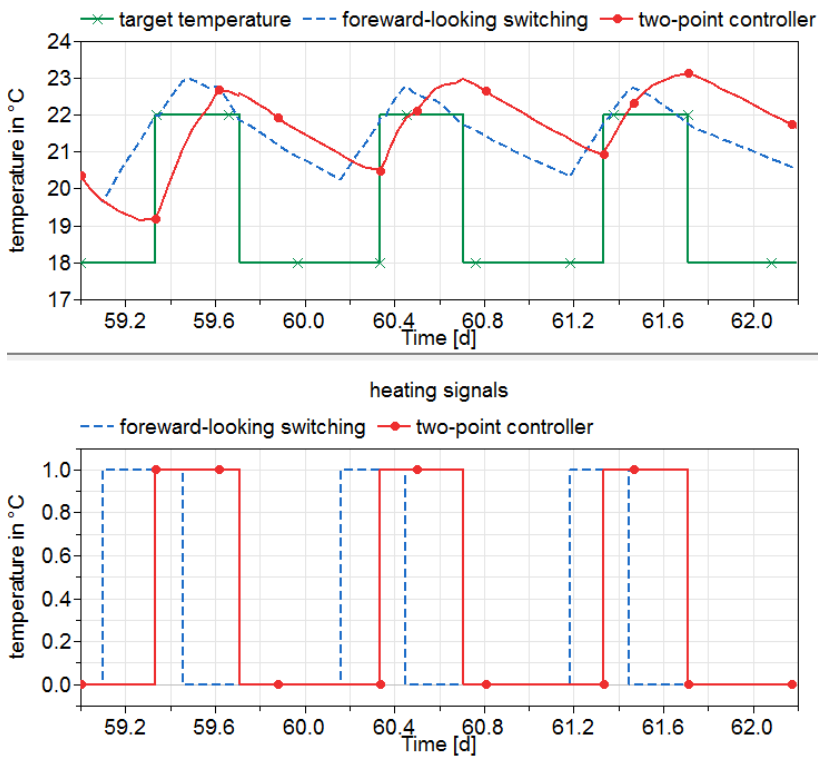

Figure 8. Room temperature and heating signal for twopoint controller and forward-looking switching
Since both of the mentioned controllers have their described properties which might be drawbacks in some cases, the two controller approaches are combined and considered as the third type of room temperature controller within this study called combi-controller.

\section{$\underline{\text { Statechart controller }}$}

Related to defined conditions (e.g. desired temperatures) certain states occur naturally (e.g. actual temperature too high or low). Those are identified as system states that require certain actions (e.g. heating) and represented as finite state machines (statechart). The statechart controller [Clauß, 2014] presented here controls the required target temperatures due to the occupancy of the room and it calculates the set points for the heating system to achieve those target temperatures. To realize those control actions two statecharts were developed that work together. Figure 9 shows one of those statecharts. It calculates the target temperatures dependent on the occupancy situation in the room

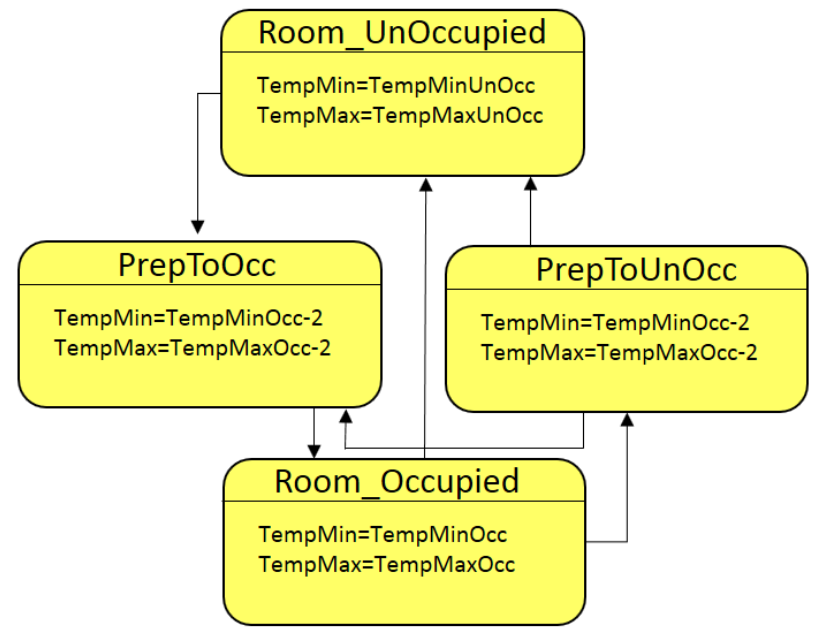

Figure 9. Occupancy statechart to calculate target temperature

The occupancy statechart contains the four states Room_Unoccupied, PrepToOcc (preparation state for oncoming occupancy), Room_Occupied and PrepToUnOcc (preparation state for oncoming leaving). Within those states different parameters are calculated, e.g. minimal (TempMin) and maximal (TempMax) temperatures and set points for heating and cooling for each state.

Values of control variables that have to be determined and decision to be taken, which are normally represented by transitions are calculated by a parameterized function approach which combines the available sensor values by a physically motivated equation. For example, the length of the preparation time (PrepTimeOcc) the room is pre-heated in order to reach the target temperature when persons enter or leave the room. With $T r$ - room temperature, Tout - ambient temperature and Tmin/Tmax - admissible minimal/ 
maximal room temperature the following heuristic and parameterized function approach to calculate the preparation time PrepTimeOcc is used

$$
\begin{aligned}
\text { PrepTimeOcc } & =c_{0} \\
& +c_{1}(\text { below }(\text { Tr }, \text { Tmin }) \\
& + \text { below }(\text { Tout }, \text { Tmin }))^{2} \\
& +c_{2}(\text { above }(\text { Tr }, \text { Tmax }) \\
& +\operatorname{above}(\text { Tout }, \text { Tmax }))^{2}
\end{aligned}
$$

with

$$
\begin{aligned}
& \text { below }(a, b)=\left\{\begin{aligned}
b-a & \text { if } a<b \\
0 & \text { else }
\end{aligned}\right. \\
& \text { above }(a, b)=\left\{\begin{aligned}
a-b & \text { if } a>b \\
0 & \text { else }
\end{aligned}\right.
\end{aligned}
$$

Equation (5) was chosen so, that the preparation time increases if the room temperature or the ambient temperature are outside the interval [T Tin, Tmax]. Other parameterized function approaches calculate the set point for the heating system respectively in the occupied or unoccupied state of the room. The parameters $c_{0}, c_{1}, c_{2}$ in (5) and other parameters of the statechart controller are determined by optimization.

The statechart controller is implemented in Modelica using if-then-else constructions.

\section{P-controller}

The fifth controller used in this study is a well-known pcontroller with limited output. It is taken from the Modelica Standard Library (Modelica.Blocks.Continuous.LimPID).

\section{Simulation Study}

The aim of the work presented in this paper is to analyze the suitability of different control strategies to ensure a desired room temperature with possibly less net energy consumption. Therefore, each room model is simulated with each controller model. To ensure the comparability of the results, for each combination of room model and controller model simulation scenarios were defined. Those guarantee the same simulation conditions e.g. ambient and boundary conditions of the room or the number of people entering the room at specified moments in time. The scenarios represent the usage of the rooms during heating periods (mid seasons, winter) since heating is the only action that can be done actively in the rooms (no cooling facilities are regarded).

In the following, one scenario is chosen to demonstrate the functioning of the controllers and further steps like their evaluation and optimization are discussed.

\subsection{Scenario "Working Period" for the office room}

This scenario was developed for the single office room. It comprises three working weeks from February 28 to March 21 including weekends. The daily working time from Monday to Friday is from 8.00 am to $5.00 \mathrm{pm}$. During this time, the desired target temperature is $22^{\circ} \mathrm{C}$. During absence of people the target temperature is 18 ${ }^{\circ} \mathrm{C}$ (Figure 10).

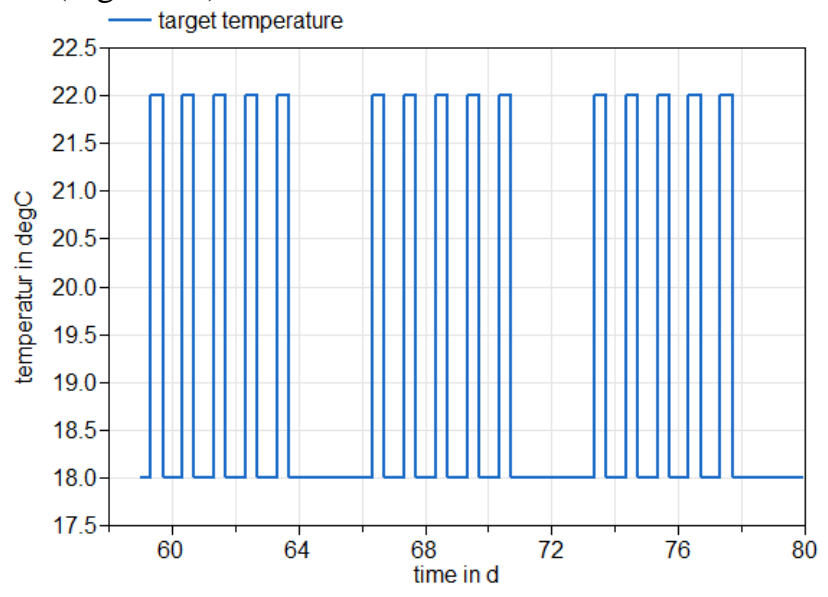

Figure 10. Target temperature of scenario "working period" for the office room

The aim of the controller models is to keep the office room temperature as close as possible to the set point while minimizing net energy consumption. To measure that the deviation between target and room temperature is calculated during occupancy and distinguished between too warm for times when the room temperature is more than $1 \mathrm{~K}$ higher than the target temperature and too cold if the room temperature is more than $1 \mathrm{~K}$ below the target temperature. To calculate the total times (too warm total and too cold total) too warm and too cold are integrated over the simulation time period. Looking at the example of the room temperature controlled by the two-point controller (Figure 11), the signal tooCold shows that at the beginning of every working day, it is too cold. Especially on Mondays, it is too cold nearly half of the day since the room temperature decreased a lot on the weekends before. The energy consumption is $159.6 \mathrm{kWh}$, the total time where it was too cold is 16.8 $\mathrm{h}$ and the total time where it was too warm in the room is $0.1 \mathrm{~h}$. 

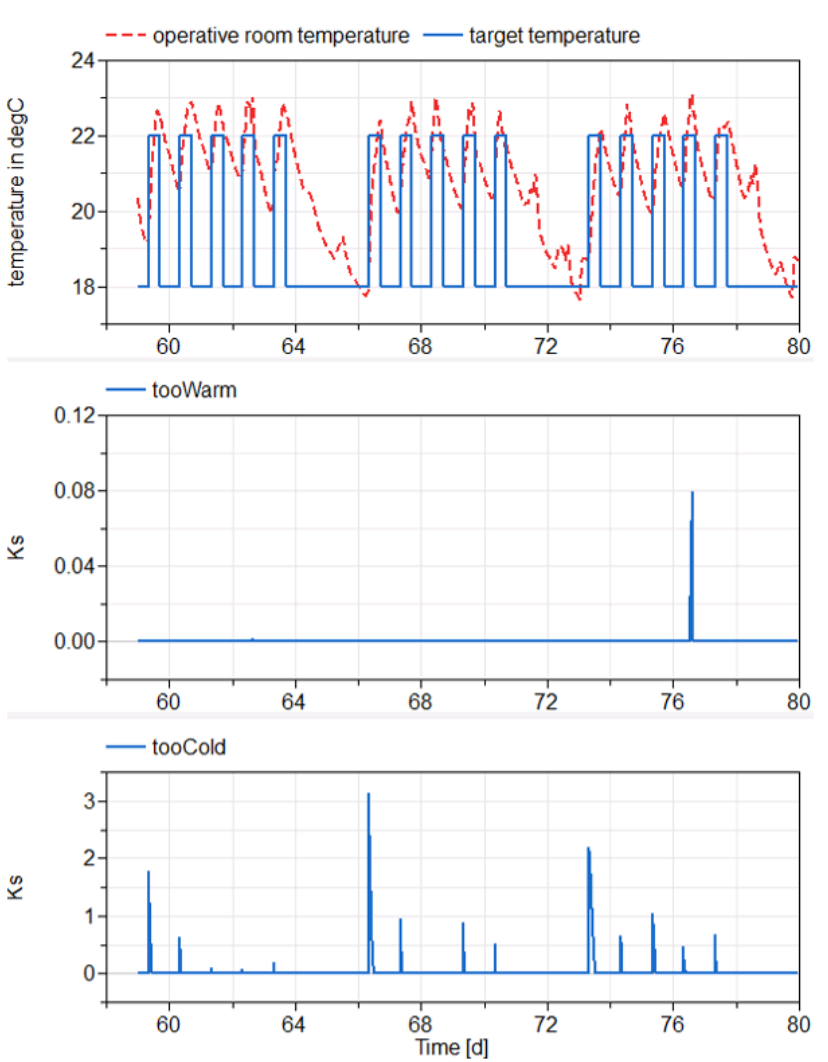

Figure 11. Room temperature and signals tooWarm and tooCold achieved by two-point controller

In comparison to the two-point controller, the statechart controller meets the required target temperature better however more net energy consumption is required. The reason for that is that before the new week begins, the room is being preheated in order to meet the high target temperature on Monday morning. Figure 12 shows the operative room temperature caused by the statechart controller as well as the signals tooWarm and tooCold. The energy consumption is $168.3 \mathrm{kWh}$ and the total time where it has been too cold is $7.4 \mathrm{~h}$. It is never too warm in the room.
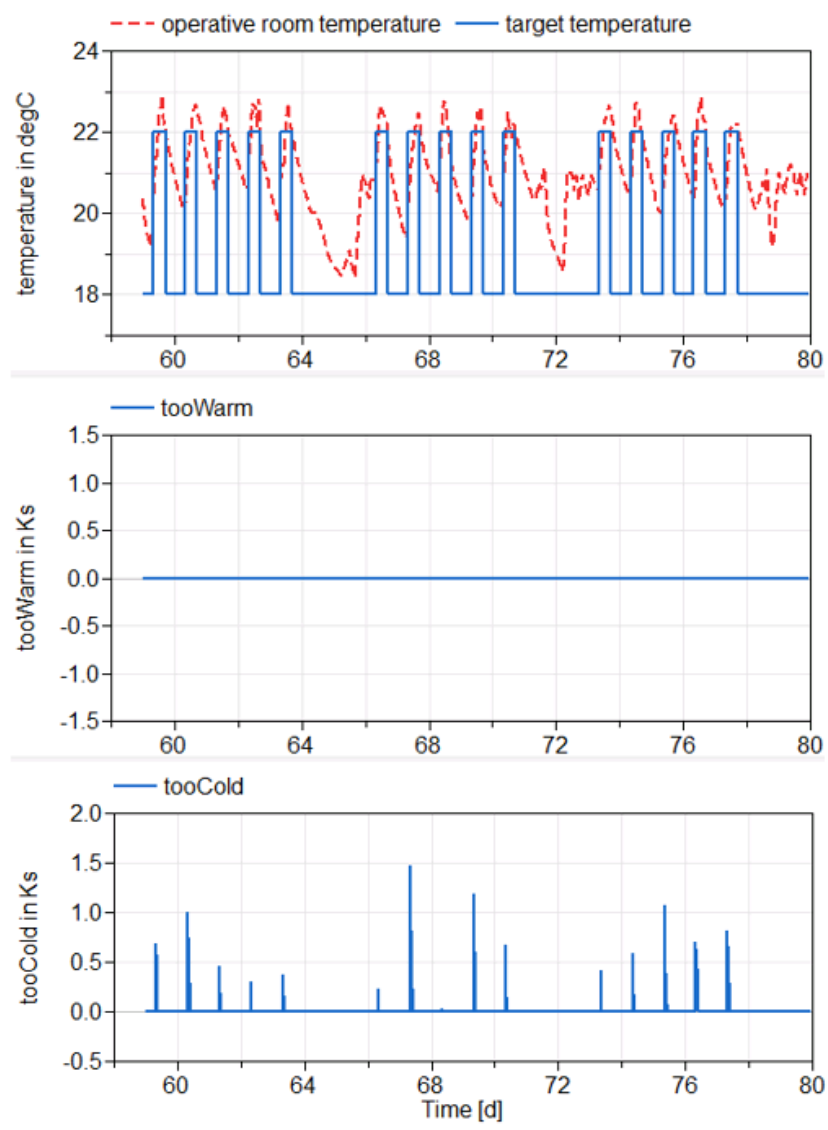

Figure 12. Room temperature and signals tooWarm and tooCold achieved by statechart controller

Table 1 shows the net energy consumption and the aggregated times when it was too warm or too cold in the room coursed by the five introduced controllers. It can be seen that the combi controller containing the forward-looking switching controller and the two-point controller, needs the most net energy, however it meets the required target temperature best whereas the pcontroller needs least energy at the price of the strongest comfort violation with regard to the times when it is too cold in the room. Figure 13 shows the room temperatures caused by the five different controllers as well as the desired target temperature

Table 1. Comparison of controllers regarding net energy consumption and violation of comfort boundaries

\begin{tabular}{|l|l|l|l|}
\hline Controller & $\begin{array}{l}\text { Energy } \\
\text { in kWh }\end{array}$ & $\begin{array}{l}\text { tooWarm } \\
\text { (total) in } \mathrm{h}\end{array}$ & $\begin{array}{l}\text { tooCold } \\
\text { (total) in } \mathrm{h}\end{array}$ \\
\hline two-point & 161.1 & 2.1 & 8.3 \\
\hline $\mathrm{FS}$ & 163.9 & 0.9 & 0 \\
\hline combi & 175.5 & 0 & 0 \\
\hline statechart & 168.3 & 0 & 7.4 \\
\hline $\mathrm{p}$ & 137.31 & 0 & 32.4 \\
\hline
\end{tabular}




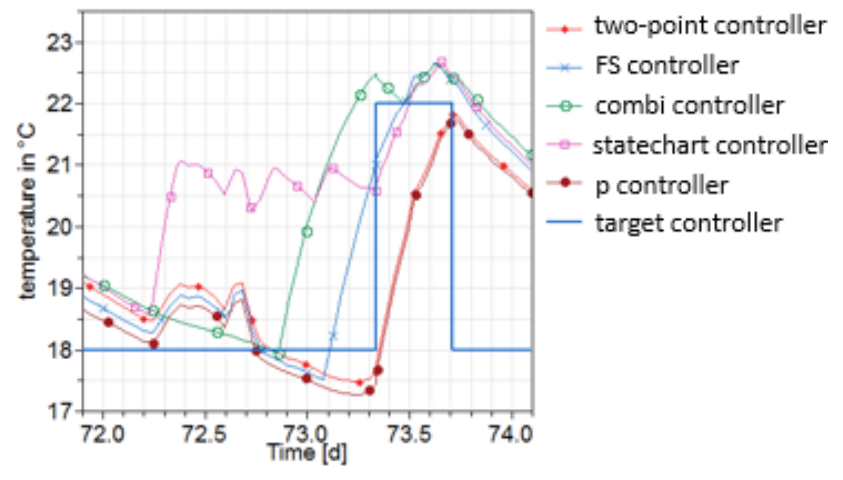

Figure 13. Room temperatures caused by the five controllers

The so far investigated eight simulation scenarios for each combination of the four room models and the five controller models show, that all of the controller models work different and fit better to one or another room models. Therefore, it is promising to take the type of the room and its HVAC equipment into consideration when choosing a suitable room temperature controller.

\subsection{Further Steps}

Up to now, the introduced controller models are parameterized from experience of the modeler. To achieve less energy consumption by sticking to the temperature comfort conditions, optimization of the controller parameters is considered. Optimization will be done by using the generic optimization program GenOpt [Wetter 2000]. GenOpt can be used with simulation programs that support textual based input/output functionality like EnergyPlus [EnergyPlus 2016], TRNSYS [TRNSYS, 2016] or Dymola [Dymola, 2016]. For the optimization, a cost function is needed that characterizes if a controller model is "good". This evaluation will be done by rating the net energy consumption and the violation of the comfort specifications regarding the room temperature. Equation (8) shows the considered cost function in principle. cost $=$ energy + penalty $1 \cdot$ tooWarmTotal + penalty $2 \cdot$ tooColdTotal

In (8) energy denotes the net energy for heating the room, tooWarmTotal and tooColdTotal are the total times the room has been to warm or to cold respectively. The two penalty terms are weighting factors.

The principal optimization procedure was so far tested for the scenario working period for the office room controlled by the two-point controller using Dymola. The two-point controller has one free parameter, the hysteresis parameter that can be tuned in order to optimize the cost function. This parameter was allowed to vary within the boundaries of 0.1 and 5 . The minimal cost function value was reached at a hysteresis parameter of 1.04. To verify this result, a parameter variation using the mos-script functionality in Dymola was operated which showed the same result. The net energy consumption decreased from $161.1 \mathrm{kWh}$ for the default hysteresis parameter value of 2 to $159.5 \mathrm{kWh}$ for the optimized hysteresis parameter value.

After optimizing the controller parameters for all scenarios, sensitivity analyses of the optimized parameters regarding different locations (including different weather) of the rooms, different HVAC systems and other, still to be defined parameters, will be performed. Aim of this analysis is to figure out how robust the controller parameters are.

The subsequent step will be to find and establish criteria to assess the quality of the controller and hence to deduce rules for choosing a specific controller to a given room and decide if the controller needs special parameter adaption or if the default parameter will be sufficient.

\section{Conclusion}

The work presented in this paper are the first steps of a broad investigation with the aim to develop a methodology to provide rules and guidelines for choosing a suitable room temperature controller with regard to the given room and its installed HVAC technology. To achieve this goal a simulation study is performed. The instrument of simulation instead of measured data is used in this study for several reasons. First, one is considerably faster than real-time. Second, the investigations can be done under specified conditions and third, several scenarios can be elaborated and easily compared to each other. This paper presents the first steps of this investigation which is the development of four different representative room models with different heating systems like floor heating, electrical heating and radiator heating as well as five controller models of important controller types. Within the simulation of defined scenarios suitable for the type of the room (e.g. office room, classroom), the eligibility of the controller models is investigated. In addition, an outlook to further steps is given which will be the optimization of controller parameters including the definition of a cost function, a sensitivity analysis to study the robustness of the optimized controller parameters and the definition of criteria to evaluate the quality of the controller.

\section{Acknowledgements}

This paper is based on the results of the research project „Entwurfsverfahren für ganzheitliche Energiemanagementsysteme in Gebäuden (ENERMAT)“, funding reference 03ET1084A.

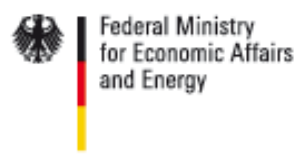

The authors are much obliged to the contributors of the research project Torsten Blochwitz, Eva Fordran, 
Matthias Franke, Jeanette Floss, Jürgen Haufe, Jörg Hohlfeld, Edgar Liebold, Richard Meyer, Paul Pinther, Stephan Seidel and Jens Wurm.

\section{References}

Abdolreza Rahmati, Farzan Rashidi, Mehran Rashidi: A hybrid fuzzy logic and PID controller for control of nonlinear HVAC systems. Proceedings of IEEE International Conference on Systems, Man and Cybernetics, pp. 2249-2254, 2003.

Abdul Afram, Farrokh Janabi-Sharifi. Theory and application of HVAC control systems - A review of model predictive control (MPC). Building and Environment 72, pp. 343-355, 2014. http://dx.doi.org/10.1016/j.buildenv.2013.11.016

Alessandra Parisio, Marco Molinari, Damiano Varagnolo, et al.: A Scenario-based Predictive Control Approach to Building HVAC Management Systems. IEEE International Conference on Automation Science and Engineering (CASE), pp. 428-435, 2013.

Christoph Clauß, Eva Fordran, Matthias Franke, et al. Entwicklung und Optimierung von Gebäude-ManagementSystemen. Fifth German-Austrian IBPSA Conference, pp.166-173, Aachen, 2014.

Christoph Nytsch-Geusen, Jörg Huber, Manuel Ljubijankic, Jörg Rädler. Modelica BuildingSystems - eine Modellbibliothek zur Simulation komplexer energietechnischer Gebäudesysteme. Bauphysik 35, Heft1, Ernst \& Sohn Verlage für Architektur und technische Wissenschaften GmbH \& Co. KG, 2013

Deutscher Wetterdienst. Testreferenzjahre von Deutschland für mittlere, extreme und zukünftige Witterungsverhältnisse. Handbuch, 2014. Website: www.dwd.de

Dymola Website: http://www.3ds.com/productsservices/catia/products/dymola

Elisa Carlon, Markus Schwarz, Christoph Schmidl, et al. Low Energy Houses Heated By Biomass Boilers: Optimization Of The Heating System Control Strategy By Means Of Dynamic Simulation. $3^{\text {rd }}$ International High Performance Buildings Conference, pp.3303/1 - 3303/8, 2014.

EnergyPlus Website: https://energyplus.net/

Frauke Oldewurtel, Alessandra Parisio, Colin N. Jones et al.: Energy efficient building climate control using stochastic model predictive control and weather predictions. Proceedings of American control conference, 2010

Friedrich Sick, Sebastian Dietz, Gustav Hillmann, Margarethe Korolkow, Susanne Rexroth. Monitoring PlusenergieGrundschule Hohen Neuendorf und IEA Task 41 (Solar Energy and Architecture). Schlussbericht, Berlin, 2015, Website:

http://www.enob.info/fileadmin/media/Publikationen/EnB au/Projektberichte/27_Projektbericht_EnBau_0327430M_ -_Monitoring_Schule_Hohen-Neuendorf.pdf

Kristin Majetta, Christoph Clauß, Jürgen Haufe, et al. Design and Optimization of an Energy Manager for an Office Building. ASIM/GI-Section Workshop - Simulation of Technical Systems \& Methods in Modeling and Simulation, pp. 289-296, 2015.

Michael Wetter. Design optimization with GenOpt. Building Energy Simulation User News 21, p. 200, 2000.
Patrick Lauenburg, Janusz Wollerstrand. Adaptive control of radiator systems for a lowest possible district heating return temperature. Energy and Building 72, pp. 132-140. 2014. http://dx.doi.org/10.1016/j.enbuild.2013.12.011

Stephan Seidel, Christoph Clauß. Eva Fordran, et al.: Design and Optimization of Building Energy Management Systems. Proceedings of $18^{\text {th }}$ ITI Symposium. pp. 329-337, November 9-11, Dresden, 2015.

Rolf Isermann. Identifikation dynamischer Systeme 1, Springer-Verlag, 2. Auflage, pp. 189-195, 1991.

TRNSYS website: http://www.trnsys.com/

Yudong Ma, Francesco Borrelli, Brandon Hencey, et al.: Model predictive control of thermal energy storage in building cooling systems. Proceedings of the $48^{\text {th }}$ IEEE conference on decision and control, pp. 392-397, Shanghai, China, 2009.

Hermann Recknagel, Eberhard Sprenger, Ernst-Rudolf Schramek.: Taschenbuch für Heizung+Klimatechnik Oldenbourg Industrieverlag GmbH; p.804, 2011/2012. 